\title{
Test of the Applicability of the Smooth Adjustment Hypothesis in Agri-product Trade of China
}

\author{
Ai Hang ${ }^{1, a,{ }^{*}, \text { Yan Wang }}{ }^{2, b}$ \\ 1School of Economics and Commerce, South China University of Technology, Guangzhou, \\ Guangdong, China \\ ${ }^{2}$ School of Economics and Commerce, South China University of Technology, Guangzhou, \\ Guangdong, China \\ a287755583@qq.com, b564388727@qq.com
}

Keywords: Agri-product Trade, Smooth Adjustment Hypothesis, Adjustment Cost.

\begin{abstract}
Adjustment cost caused by the trade liberalization would affect countries' trade structure and industrial development, With the appearance of intra-industry trade phenomenon, 'Smooth Adjustment Hypothesis' about trade adjustment cost is taken more seriously. Agriculture always was the most basic industry in China and agricultural products occupied a large proportion in external trade. By theoretically discussing the applicability of smooth adjustment hypothesis in Chinese agricultural products trade and analyzing the applicability by Parameter estimation and non-Parameter estimation, we found that, Smooth adjustment hypothesis was partly suitable for Chinese agricultural products trade, and it needs three years when dynamic change of intra-industry trade had a significant negative relationship with Adjustment costs.
\end{abstract}

\section{“平滑调整假说”在我国农产品贸易中的适用性检验 \\ 杭爱 $1,{ }^{,}{ }^{*}$, 王彦 $2, \mathrm{~b},{ }^{*}$ \\ 1华南理工大学经济与贸易学院, 广州, 广东, 中国 \\ 2华南理工大学经济与贸易学院, 广州, 广东, 中国 \\ a287755583@qq.com, b564388727@qq.com}

关键词: 农产品贸易; 平滑调整假说; 调整成本

中文摘要. 贸易自由化引致的调整成本会影响各国贸易结构和产业发展, 随着产业内贸易现 象的出现，有关贸易调整成本的 “平滑调整假说” 越来越受到重视。农业是我国基础性产业， 农产品在对外贸易中占有重要地位。本文从理论上探讨了 “平滑调整假说” 在我国农产品贸 易中的适用性, 并通过参数估计和非参数估计两种方法实证分析了适用性问题。结果显示: “平滑调整假说” 在一定程度上适用于我国农产品贸易的结论, 然而动态的产业内贸易变化 与调整成本需要三年的时间才能显著表现出负向关系。

\section{1. 引言}

随着全球贸易自由化程度的提升, 贸易引致的调整成本正影响着各国的产业结构, 有关 贸易调整成本的 “平滑调整假说” 越来越受到重视。1966年Balassa提出 “平滑调整假说”

(Smooth Adjustment Hypothesis), 认为在贸易自由化的背景下, 生产要素的调整会随着产 业内贸易水平的提升而更多地在产业内进行调整, 从而减少了调整成本 ${ }^{[1]}$ 。此后对于该假说 
的研究多集中于工业制造业以及工业化发达国家, 却较少涉及农业。与此同时, 对产业内贸 易理论的研究越来越多，但基于调整成本的研究还较少。

我国在加入WTO后，贸易开放程度逐渐加大，各项贸易额都有较大幅度增长，因而研究 贸易调整成本对促进我国贸易发展、促进产业结构调整具有重要意义。农业作为我国的基础 产业，在加入WTO后必然会受到激烈竞争的背景下，如何有效地提高农产品竞争力、调整农 业生产结构事关国计民生。

因此本文希望通过验证 “平滑调整假说”是否存在于我国农产品贸易中，将“平滑调整 假说” 拓展到农产品贸易领域。在此基础上, 我们可以测算出历年我国农产品对外贸易调整 成本, 从产业内贸易角度研究农产品生产结构, 并对我国发展农产品产业内贸易提出建议。

\section{2. 文献综述}

“平滑调整假说” 认为在贸易自由化的趋势下，生产部门的生产会发生变化，导致要素 在部门间流动, 要素市场如不能及时地对供需变化做出调整, 就会产生调整成本, 而要素在 同一部门内部重新分配产生的成本小于部门间产生的调整成本。关于 “平滑调整假说” 的理 论研究相对较少。Neary（1985）[2]认为部门间非完全替代的劳动力和刚性名义工资是导致调 整成本的两个主要因素, 因此应该从这两个方面着手促进产业内贸易发展, 进而减少贸易产 生的调整成本。Brülhart和 Elliott（2002）[3]研究了不同条件下的调整成本，并通过 Jones-Samuelson特定要素模型发现工资差别和失业是调整成本的两个表现形式。Lovely和 Nelson（2000） ${ }^{[4]}$ 认为如果存在规模经济, 产品种类会减少而产量规模性增加, 因而高水平的 产业内贸易也许只是一种假象, 实质是产业间调整。二人于 2002 年提出 “平滑调整假说” 成 立的前提是国内吸收不变 ${ }^{[5]}$ 。在实证研究方面, Brülhart (2000) [6]构造了包括显性需求变化、 贸易开放程度、外资等变量的多变量模型，采用了爱尔兰的宏观面板数据验证 “平滑调整假 说” 并进行了影响因素分析, 结论支持假说, 但不支持调整成本主要受到产业内贸易的影响。 Cabral和Silva（2006） [7]从微观的角度, 采用葡萄牙家庭个人数据进行研究, 结论也支持 “平 滑调整假说”。杜运苏和郭羽诞（2009） [8]采用1987-1999年我国制造业面板数据进行了贸易 调整成本和产业内贸易关系的研究, 得出了产业内贸易导致的经济调整成本低于产业间贸易 的结论。于峰和卢进勇 (2012) [9]利用多元非参数回归模型证明了 “平滑调整假说” 存在于 我国农产品贸易中。然而, 并不是所有实证研究均支持 “平滑调整假说”。Brülhart和Thorpe （2000） [10]运用1970至1994年马来西亚的面板数据模型分析了产业内贸易与劳动力调整成本 的关系，认为二者并非呈负向关系。Greenaway、Haynes和Milner（2002） ${ }^{[11]}$ 在运用英国工业 数据进行研究时同样否定了假说的存在, 他们认为调整成本的代理变量是产业层面或部门层 面的工人人数变化, 而劳动力的变化包括资源和非自愿失业, 如果自愿失业过多就会对结果 产生影响。郭东杰、郡琼燕（2012） [12]通过对我国制造业进行分析发现，产业内贸易对劳动 力调整的影响存在一定滞后, 且产业内贸易水平增加反而导致劳动力在产业间调整, 结论不 支持 “平滑调整假说”。

已有研究表明, 产业内贸易、劳动力调整水平等变量的选取决定了 “平滑调整假说” 是 否成立，同时对不同国家、行业的选取也具有重要影响。基于此，本文采用参数估计法论证 “平滑调整假说” 在我国农产品贸易中的适用性。同时考虑到论证的完整性, 针对模型设定 误差可能引起假说不成立的情况, 本文还采用了非参数估计的方法, 对产业内贸易水平与劳 动力调整间的关系进行再一次的验证。 


\section{3. 模型、数据与变量}

\section{1 模型设定与指标选取}

假设某一产品的生产函数为 $Y=A^{\gamma} K^{\theta} L^{1-\theta}$, 其中Y、A、 $\mathrm{K} 、 \mathrm{~L}$ 分别代表产出、生产技术、 资本存量以及劳动力数量。根据企业利润最大化的一阶条件, 一是资本的边际收入等于资本 价格 $\mathrm{r}$, 二是劳动的边际收入等于工资 $\mathrm{w}$ 。在此基础上, 我们可以将生产函数推导成为 $Y=A^{\gamma}\left(\frac{\theta}{1-\theta}\right)^{\theta}\left(\frac{w}{r}\right)^{\theta} L$, 在等式两边取对数, 整理得到劳动需求的表达式:

$$
\ln L=-\gamma \ln A-\theta \ln \left(\frac{w}{c}\right)+\ln Y
$$

式 (1) 为基本模型, 由于相关的贸易理论没有给定劳动力市场调整成本的分析模型, 也 没有说明对劳动力市场调整进行分析时应该包含哪些变量, 本文参照Brülhart和Thorpe (2000) 文章的模型, 并结合已有研究, 选择五个指标作为计量模型的变量。根据上述分析, 设定参 数估计法的计量模型如下:

$$
\ln \left|D E M P L_{t}\right|=c+\beta_{1} \ln \left|D A D_{t}\right|+\beta_{2} \ln \left|D I N C O M E_{t}\right|+\beta_{3} M I I T_{t}+\beta_{4} \ln M T R E X_{t}+\varepsilon_{t}
$$

其中, 农业劳动力调整成本 (DEMPL) 作为调整成本的代理变量, 本文以第一产业第 年就业人数相对变化量代替; 农产品显性需求变化 (DAD) 表达式为 $\mathrm{Y}_{\mathrm{t}} \mathrm{X}_{\mathrm{t}}+\mathrm{M}_{\mathrm{t}}$, 其中 $\mathrm{Y}_{\mathrm{t}} 、 \mathrm{X}_{\mathrm{t}}$ 、 $\mathrm{M}_{\mathrm{t}}$ 分别表示农产品第 $\mathrm{t}$ 年产值、出口额和进口额; 农业劳动者人均收入变化（DINCOME）采 用人均年纯收入指标的变化量来表示; 采用A指数来表征边际产业内贸易（MIIT）, 表达式 为 $\mathrm{A}_{\mathrm{i}}=1-\left|\Delta \mathrm{X}_{\mathrm{i}}-\Delta \mathrm{M}_{\mathrm{i}}\right| /\left(\left|\Delta \mathrm{X}_{\mathrm{i}}\right|+\left|\Delta \mathrm{M}_{\mathrm{i}}\right|\right)$, 其中 $\mathrm{Ai}$ 表示某种农产品的 $\mathrm{A}$ 指数, $\Delta \mathrm{X}_{\mathrm{i}}$ 和 $\Delta \mathrm{M}_{\mathrm{i}}$ 分别表示两阶段 农产品的出口和进口额的变化量; 贸易开放度 (MTREX) 表达式为 $\frac{\left(\mathrm{X}_{\mathrm{t} 1}+\mathrm{M}_{\mathrm{t}}\right)+\left(\mathrm{X}_{\mathrm{t}^{2}}+\mathrm{M}_{\mathrm{t} 2}\right)}{\mathrm{Y}_{\mathrm{t} 1}+\mathrm{Y}_{\mathrm{t} 2}}$ 。

考虑到产品需求将吸引更多劳动力从事生产活动, 因此我们预测 DAD参数符号为正; 在 劳动力正常流出的情况下, 收入增加可能吸引更多的劳动力进入农业部门, DINCOME的参 数符号未定; “平滑调整假说” 认为产业内贸易的发展会减少调整成本, 预测MIIT的参数符 号为负; 一般认为贸易会加剧产品竞争, 从而使得部门间劳动力流动更大, 预测该变量参数 符号为正。

\section{2 数据说明}

考虑变量所需数据统计口径的一致性, 本章各变量所采用的数据均来源于 《中国统计年 鉴》, 涵盖1980年至2011年的数据。关于贸易商品的分类，统计年鉴中有SITC和HS两种分类 法, 后者较前者包含的商品范围更广, 但由于其数据的年份较近, 无法满足本章研究所需的 数据需求, 因而本章选择统计年鉴中SITC Rev.3 (《国际贸易标准分类》第3次修订标准)分类 数据。根据SITC Rev.3分类，农产品的界定如表1所示。

表1 SITC分类农产品

\begin{tabular}{|c|c|}
\hline SITC分类 & 农产品名称 \\
\hline 0类 $(00-09$ 章 $)$ & 食品及活动物 \\
\hline 1类 (11-12章 $)$ & 饮料及烟类 \\
\hline 2类（21-23章, 26-29章 $)$ & 非食用原料（燃料除外） \\
\hline 4类 (41-43章) & 动植物油、脂及蜡燃料 \\
\hline
\end{tabular}

本文在计算农产品产业内贸易水平时采用了加总的农产品贸易额。此外, 由于各变量原 始数据单位不统一并且考虑物价变动等影响, 需要预先对所有数据进行处理, 按照各年汇率 将单位由美元转换成人民币, 并通过居民消费价格指数进行数据调整。 


\section{4. 实证结果及分析}

\section{1 参数估计法}

本文的参数估计法通过对时间序列数据采取协整分析来进行。根据已有研究，本文对需 要做差分的变量分别间隔一年、两年和三年进行差分，并根据检验结果（表2）将间隔一年的 变量序列中的InMTREX和间隔三年的变量序列中的InMTREX进行针对性修改, 从而进行协整 检验。

表2 单位根检验结果

\begin{tabular}{|c|c|c|c|c|c|c|c|}
\hline \multirow{2}{*}{ 变量 } & \multicolumn{2}{|c|}{ 间隔一年 } & \multicolumn{2}{c|}{ 间隔两年 } & \multicolumn{3}{c|}{ 间隔三年 } \\
\cline { 2 - 8 } & 系数 & 一阶差分 & 系数 & 一阶差分 & 系数 & 一阶差分 & 二阶差分 \\
\hline $\ln |\mathrm{DEMPL}|$ & $-4.14271^{* *}$ & - & $-3.51624 *$ & - & -2.71625 & $\begin{array}{c}-5.09095 * * \\
*\end{array}$ & - \\
\hline $\ln |\mathrm{DAD}|$ & $\begin{array}{c}-5.40308^{* *} \\
*\end{array}$ & - & $-3.56618^{*}$ & - & -3.00918 & $\begin{array}{c}-4.48072 * * \\
*\end{array}$ & - \\
\hline $\ln |\mathrm{DINCOME}|$ & $-4.11461 * *$ & - & $-3.67117 * *$ & - & -1.69521 & $\begin{array}{c}-5.30411^{* *} \\
*\end{array}$ & - \\
\hline $\mathrm{MIIT}$ & $\begin{array}{c}-5.53139^{* *} \\
*\end{array}$ & - & $-4.00315^{* *}$ & - & -1.21932 & $-4.30158 * *$ & - \\
\hline $\ln \mathrm{MTREX}$ & -2.43211 & $-3.40031^{*}$ & $-2.45762 * *$ & - & -2.17246 & -1.18773 & $\begin{array}{c}-10.68553 * \\
* *\end{array}$ \\
\hline
\end{tabular}

注: ***、**、*分别表示在1\%、5\%、10\%的显著性水平下通过检验

为了检验单整序列间是否存在长期的稳定关系，本文分别对间隔期为一年、两年和三年 的时间序列进行回归分析, 结果见表3。从协整检验的结果可以大致看出, 间隔期一年和间隔 期三年的回归结果最理想，均只有一个变量的回归系数不显著，而间隔期两年的回归结果则 有多个变量的系数不显著, 因而可以视为回归结果不理想。对残差单位根检验和怀特检验的 结果（表4）表明, 三个模型的残差均通过了单位根检验, 表明残差序列平稳, 即三个方程的 变量间均存在长期稳定关系。怀特检验的结果均拒绝了原假设，表明三个方程均无异方差。 表3 三种时间序列协整检验的回归结果

\begin{tabular}{|c|c|c|c|}
\hline & \multicolumn{3}{|c|}{ 被解释变量为 $\ln |\mathrm{DEMPL}|$} \\
\hline & 间隔一年 & 间隔两年 & 间隔三年 \\
\hline 常数项 & $21.10591 * *$ & $17.56237 * * *$ & $3.460162^{* *}$ \\
\hline $\ln |\mathrm{DAD}|$ 系数 & $-2.218596^{* * * *}$ & -0.655893 & $1.266475^{* * *}$ \\
\hline $\ln \mid \mathrm{DINCOME|}$ 系数 & $0.364481^{* * *}$ & 0.424249 & $-0.973168^{* * *}$ \\
\hline MIIT系数 & 1.334643 & 1.336576 & $-2.938314^{* * *}$ \\
\hline $\operatorname{lnMTREX}$ 系数 & - 一 & $3.597379 * * *$ & - \\
\hline DlnMTREX系数 & $3.954670^{* * *}$ & - & -2.814007 \\
\hline $\mathrm{R}^{2}$ & 0.498449 & 0.363103 & 0.501688 \\
\hline F值 & $6.459805^{* * *}$ & $3.420676^{* *}$ & $5.788956^{* * *}$ \\
\hline
\end{tabular}

注: ***、**、*分别表示在 $1 \% 、 5 \% 、 10 \%$ 的显著性水平下通过检验 
表4 残差单位根检验和怀特检验结果

\begin{tabular}{|c|c|c|c|}
\hline & 间隔一年 & 间隔两年 & 间隔三年 \\
\hline $\mathrm{ADF}$ 统计量 & $-5.301701 * * *$ & $-3.765380 * *$ & $-4.077194 * * *$ \\
\hline $\mathrm{N} * \mathrm{R}^{2}$ & 9.921517 & 19.97253 & 16.76665 \\
\hline
\end{tabular}

注: ***、**、*分别表示在1\%、5\%、10\%的显著性水平下拒绝原假设

由于协整检验的目的仅为验证二者存在的关系，而非为了预测，因而我们只需关注各项 系数的符号及显著性, 并考虑模型方程的设置合理性。

（1）参看间隔期为两年的检验结果，其中有三个变量的 $\mathrm{T}$ 检验不显著，且在估计出来的 系数符号中, 显性需求变化、边际产业内贸易指数的系数符号与前文预测不同, 说明显性需 求DAD在两年的时间段内对劳动力调整的影响并不大，且产业内贸易水平MIIT的变化也并没 有与劳动力调整负向变动。考虑到间隔两年期的 $\mathrm{R}^{2}$ 值小于其他两个时期, 表明在一到两年期 间本文设定的模型中选取的几个变量对劳动力调整影响有限。

（2）在间隔期为一年的模型中， $\mathrm{R}^{2}$ 值较间隔期为两年的模型有了一定增加，且只有一个 变量, 即边际产业内贸易指数MIIT的系数不显著, 相对应的F值表明这些变量在一年时间内 对劳动力变动有显著影响。与前文预测不同, 显性需求DAD的系数显著为负, 表面意义为国 内需求的变化越大, 劳动力调整成本越小, 即劳动力流动不明显。对于该现象, 我们可以解 释为劳动力调整在短时间内存在一定障碍, 因而劳动力不能及时应对市场需求以调整自身农 产品的生产。在农业生产中, 这种现象具体表现为某些农作物生产周期长, 因此尽管市场需 求已经产生变动，但这些农作物的生产者不能短时间内放弃生产。

（3）在间隔期为三年的模型中， $\mathrm{R}^{2}$ 值大于其他两个时期，同样其中也有一个变量贸易开 放度MTREX的系数不显著, 相对应的F值表明这些变量在三年时间内对劳动力变动有显著影 响。在该模型中, 边际产业内贸易MIIT显著地与劳动力调整成本成负向关系, 即农产品产业 内贸易水平越高, 农业的劳动力变化越小, 因为在长期时间里, 劳动力可以在产业内进行其 他农作物的生产, 平滑调整假说在此时成立。同时, 显性需求DAD的系数也与前文预测相同, 表现为与劳动力调整正向变动, 这表明在长期时间里, 如果对农产品的需求非常旺盛, 会引 致更多的劳动者进入农业生产，从而使得劳动力流动增大。与间隔一年的模型相比，收入变 动DINCOME的系数明显有异, 表现为显著性为负, 即收入变动与农业的劳动力变动相反。 可能的解释为我国大量农村青年进城务工, 他们的眼光更长远, 农业劳动者暂时性的收入增 加并不会吸引他们, 留在农村的多数为老年人, 这一部分老农民不会因为收入的增加或减少 而放弃耕作。相反, 由于农业是一个赢利少、风险大的基础产业, 在长期时间内, 理性的企 业并不会盲目地因为暂时性收入增多而选择进入, 从而使得劳动力变化并不明显。贸易开放 度MTREX的系数在间隔三年的模型中尽管为正, 但并不显著, 说明在长期时间内, 贸易开放 度不会显著影响劳动力的调整。

结合MTREX与MIIT在三个模型中的系数符号和显著性，可以推测二者存在一定的交叉 影响, 产业内贸易作为贸易中的一部分, 整个贸易对劳动力变动的影响会在一定程度上涉及 到产业内贸易的影响。

综上所述，平滑调整假说短期内并不明显存在于我国农产品贸易中，但在长时间的跨度 里, 其在我国农产品贸易中具有一定适用性, 农产品产业内贸易水平增高, 至少需要三年时 间才能明显地表现出降低劳动力变动。综合时间间隔为一年、两年和三年的三个模型进行对 比可以发现, 显性需求、农民收入、产业内贸易水平和贸易开放程度与劳动力调整的关系会 随着时间长短在逐步改变, 而间隔期为两年时, 这些变量的变动对劳动力调整的影响最小。

\section{2 非参数估计法}

参数估计法假设变量间是线性或可转化为线性的非线性关系，而在传统的平滑调整假说 理论中，并没有足够的理论说明产业内贸易与调整成本间的关系是线性或可转化为线性的。 
因此本文采用Spearman秩相关系数法进行非参数估计以补充回归模型的缺陷部分。具体来说, 如果平滑调整假说成立, 则MIIT越大, 产业内贸易水平越高, 劳动力变动DEMPL就应该越小, 而相反地, MIIT越小, 则产业内贸易水平越低, 产业间贸易占主要部分, 劳动力调整主要发 生在产业间，因而产业内的劳动力变化DEMPL应该越大，故秩相关系数应该为负。

表5 间隔一年的Spearman秩相关系数

\begin{tabular}{|c|c|c|c|}
\hline & & $\ln \mid$ DEMPL $\mid$ & MIIT \\
\hline \multirow{2}{*}{$\ln |\mathrm{DEMPL}|$} & 相关系数 & 1.000 & -0.226 \\
\hline & $\mathrm{P}$ 值 & - & 0.221 \\
\hline \multirow{2}{*}{ MIIT } & 相关系数 & -0.226 & 1.000 \\
\hline & $\mathrm{P}$ 值 & 0.221 & - \\
\hline \multicolumn{4}{|c|}{ 表6 间隔两年的Spearman秩相关系数 } \\
\hline & & $\ln \mid$ DEMPL| & MIIT \\
\hline \multirow{2}{*}{$\ln |\mathrm{DEMPL}|$} & 相关系数 & 1.000 & -0.087 \\
\hline & $\mathrm{P}$ 值 & - & 0.649 \\
\hline \multirow{2}{*}{ MIIT } & 相关系数 & -0.08754 & 1.000 \\
\hline & P值 & 0.649 & - \\
\hline \multicolumn{4}{|c|}{ 表7 间隔三年的Spearman秩相关系数 } \\
\hline & & $\ln \mid$ DEMPL| & MIIT \\
\hline \multirow{2}{*}{$\ln |\mathrm{DEMPL}|$} & 相关系数 & 1.000 & -0.354 \\
\hline & $\mathrm{P}$ 值 & $\bar{C}$ & 0.030 \\
\hline \multirow{2}{*}{ MIIT } & 相关系数 & -0.354 & 1.000 \\
\hline & $\mathrm{P}$ 值 & 0.030 & - \\
\hline
\end{tabular}

在间隔期为一年和两年的分析中，尽管两个变量间的秩相关系数均为负，但 P 值远大于 $10 \%$ 的显著性水平，说明边际产业内贸易水平与劳动力调整间的关系虽然是负向的，但这种 关系并不显著, 此结论也与参数估计方法中的结论相同。而在间隔期为三年的秩相关系数分 析中，边际产业内贸易与劳动力调整变化量的相关系数为负，且 $\mathrm{P}$ 值为 0.03 , 说明这种负向 关系在 5\%的水平下是显著的。故我们可以认为, 在时间跨度为三年时, 产业内贸易与劳动力 调整成本有明显的负向关系, 即平滑调整假说需要三年的滞后期才能在我国的农产品贸易中 成立。

\section{5. 研究结论}

本文借鉴前人研究经验，对我国农产品贸易的相关时间序列数据进行了协整分析和 Spearman秩相关系数分析, 得出如下结论:

第一, 以劳动力调整为观察变量, 平滑调整假说在我国农产品贸易中存在一定的适用性, 但这种关系需要有一定的时间滞后才能表现出来。具体表现为短期内产业内贸易水平的提高 并不会显著影响劳动力的调整变化; 而在至少三年的长期内, 劳动力调整依然表现为产业内 流动为主, 即产业内贸易水平的提高, 确实会减少产业内劳动力的变动。因而, 可以认为平 滑调整假说在较长时期内适用于我国农产品贸易。

第二，国内农产品显性需求的变化对劳动力的影响存在从短期到长期由显著负向转变为 显著正向作用的现象。短期内, 劳动力调整存在一定障碍, 无法及时应对市场需求以调整农 产品生产, 从而造成劳动力变化不大。而在较长时间里, 如果农产品需求非常旺盛, 将会引 致更多的劳动者进入农业生产，从而使得劳动力流动增大。

第三，收入水平的变化与劳动力调整间存在不确定关系，短期内表现为显著正向关系， 长期则表现出明显的负向关系。可能的原因是短期内收入的增长确实会吸引部分劳动力投入 到农业生产中, 然而理性的人和企业都会考虑长远收入, 从而使得长期的劳动力流动并不明 显。 
最后, 贸易开放度与产业内贸易存在一定程度的交叉影响, 这是由于产业内贸易也是贸 易开放的一部分。短期内, 贸易开放度对劳动力变化的影响更为明显, 即贸易开放程度越高, 劳动力在产业间流动越大，而在长期产业内贸易对劳动力调整的负向影响更大。

\section{References}

[1] Balassa B., Tariff Reductions and Trade in Manufatures among the Industrial Countries[J]. The American Economic Review, 1966, 56(3): 466-473.

[2] Neary J.P., Theory and Policy of Adjustment in an Open Economy. In Greenaway D.(Ed.), Current Issues in International[M]. London: Macmillan, 1985, (2): 239-243.

[3] Brülhart M., and Elliott R.J.R., Labour Market Effects of Intra Industry Trade: Evidence for the United Kingdom[J]. Weltwirtschaftliches Archiv, Review of World Economics, 2002, 138(2):207-228.

[4] Lovely M., and Nelson D., On the Economic Relationship between Marginal Intra-Industry Trade and Labour Adjustment in a Division of Labour Model[J]. Review of International Ecnomics, 2000, 8(3): 436-447.

[5] Lovely M., and Nelson D., Intra-Industry Trade as an Indicator of Labor Market Adjustment[J]. Review of World Economics, 2002, 138(2): 179-207.

[6] Brülhart M., Dynamics of Intra-Industry Trade and Labor-Market Adjustment[J]. Review of International Economics, 2000, 8(3): 420-435.

[7] Cabral M. and Silva J., Intra-industry Trade Expansion and Reallocation between Sectors and Occupations[J]. Weltwirtschaftliches Arhiv. Review of world economics, 2006, 142(3): 547-567.

[8] Du Yunsu and Guo Yudant, Intra-Industry Trade and Trade-Induced Cost - Empirical Analysis Based on China's Manufacture Industry, Industrial Economics Research, 2009, (3): 9-13.

[9] Yu Feng and Lu Jinyong, Is "Smooth Adjustment Hypothesis" Applicable to Chinese Agricultural Product Trade - From Multivariate Nonparametric Regression Model Test, Journal of Central University of Finance \& Economics, 2012, (10): 62-66.

[10]Brülhart M. and Thorpe M., Intra-Industry Trade and Adjustment in Malaysia: Puzzling Evidence[J]. Applied Economics Letters, 2000, 7(11): 729-733.

[11]Greenaway D., Haynes M., and Milner C., Adjustment, Employment Characteristics and Intra-Industry Trade[J]. Review of World Economics, 2002, 138(2): 254-276.

[12]Guo Dongjie and Shao Qiongyan, Analysis of The Influences of Intra-Industry on the Labor Market Adjustment: A SAH Test Based on the Chinese Manufacturing Panel Data, Journal of International Trade, 2012, (6): 18-31. 